\title{
Faciliter l'interaction orale en anglais pour sciences humaines et sociales : la revue de presse
}

\section{Bénédicte Reyssat}

\section{OpenEdition}

1 Journals

Édition électronique

URL : http://journals.openedition.org/asp/5594

DOI : 10.4000/asp.5594

ISSN : 2108-6354

Éditeur

Groupe d'étude et de recherche en anglais de spécialité

\section{Édition imprimée}

Date de publication : 1 novembre 2018

Pagination : 190-196

ISSN : 1246-8185

\section{Référence électronique}

Bénédicte Reyssat, « Faciliter l'interaction orale en anglais pour sciences humaines et sociales : la revue de presse », ASp [En ligne], 74 | 2018, mis en ligne le 01 novembre 2018, consulté le 01 novembre 2020. URL : http://journals.openedition.org/asp/5594; DOI : https://doi.org/10.4000/asp. 5594

Ce document a été généré automatiquement le 1 novembre 2020.

Tous droits réservés 


\title{
Faciliter l'interaction orale en anglais pour sciences humaines et sociales : la revue de presse
}

\author{
Bénédicte Reyssat
}

Dans ce compte rendu d'expérience de pratique pédagogique en centre de langues, Bénédicte Reyssat nous montre à quel point une activité pédagogique d'apparence anodine, une revue de presse réalisée à l'oral par des étudiants en binôme, peut apporter des réponses concrètes à des questions directement liées à l'enseignement de l'anglais dans le domaine des sciences humaines et sociales (SHS). La première tient à la nature même de ce domaine qui, parce qu'il porte sur des objets très divers, suppose l'acquisition d'un vocabulaire à la fois large, précis et nuancé, sur des sujets parfois sensibles. La seconde réponse relève de l'ergonomie du cours d'anglais de spécialité. Dans l'activité présentée par l'auteur, l'espace est vécu de telle manière qu'il atténue fortement la pression sur les étudiants, ce qui libère la parole et augmente la pratique linguistique. Cette ergonomie nous paraît particulièrement pertinente en SHS où les lacunes culturelles des interlocuteurs sont souvent perçues comme suspectes et peuvent générer des réflexes contreproductifs de mise en retrait des échanges.

Ce compte rendu contribue pleinement aux réflexions en cours sur l'apprentissage des langues de spécialité dans le domaine SHS, notamment le cycle de journées d'études qui portent sur ce domaine émergent de l'ASP (Toulouse 2016, Chambéry 2017, Lyon 2018). Il fait également écho aux réflexions menées par la revue Recherche et Pratiques Pédagogiques en Langues de Spécialité dont le prochain numéro est consacré aux rapports entre le corps, la voix et l'enseignement-apprentissage des langues dans l'enseignement supérieur. (Philippe Millot)

\section{Contexte}

1 Peut-on acquérir une parole authentique dans une langue étrangère en restant constamment assis derrière une table et sans choisir ce dont on parle? De ce questionnement pédagogique est née l'activité proposée ci-dessous. 
2 La revue de presse est une activité pour cours LANSAD en présentiel au niveau licence actuellement en place de la L1 à la L3 pour les étudiants de sciences humaines et sociales (SHS). Elle est également utilisable en master. La poursuite sur plusieurs semestres est nécessaire et bénéfique, mais au-delà de 3 semestres il est préférable de passer à d'autres activités de type débats pour éviter l'usure pédagogique. Les enseignements de LANSAD sont délivrés du S2 au S6 de licence avec $24 \mathrm{~h}$ par semestre, puis sur les semestres 1,2 et 3 de master, avec $18 \mathrm{~h}$ par semestre. À partir du semestre 4 de licence, les étudiants suivent leurs cours dans un espace langues, le Centre Polyglotte, qui alterne des semaines en Centre de Langues et des semaines en salle banalisée, les contenus étant mis à disposition sur Moodle. Le Centre Polyglotte, ouvert en 2006, a été conçu pour faciliter les travaux de groupe, l'aide individualisée, la conversation et la personnalisation des supports.

Conçue pour une préparation au concours d'entrée des IEP adossée à la L1 d'histoire afin d'aborder la lecture d'articles d'actualité, cette activité a été systématisée pour les filières SHS afin de remédier à la passivité avec laquelle les étudiants répondent à des cours entièrement dirigés et impulsés par l'enseignant. Elle a été testée avec des groupes de 18 à 30 étudiants.

4 Elle dure de 20 à 30 minutes en début de chaque cours, sur 10 semaines et sur plusieurs semestres. Elle occupe donc environ $20 \%$ du temps de cours en licence.

\section{Objectifs}

- dynamiser le début des séances et le ritualiser ;

- diversifier les thèmes du cours et ainsi le lexique ;

- banaliser la prise de parole en continu.

\section{Organisation}

5 La classe est divisée en deux groupes, qui préparent en semaine alternée. Chaque étudiant du groupe concerné choisit un article de presse $^{1}$ et le prépare; il/elle le présente à des étudiants de l'autre groupe lors d'un travail en binôme en début du cours. Les paires permutent trois fois et les notes écrites sont interdites lors de la $3^{\mathrm{e}}$ répétition. L'enseignant répond aux questions éventuelles, qui portent fréquemment sur le lexique et les tournures. Il est à noter que l'enseignant est souvent appelé pour arbitrer un désaccord linguistique au sein du binôme.

6 La revue de presse est systématiquement évaluée par "sondage ", c'est-à-dire une écoute silencieuse par l'enseignant pendant environ 30 secondes, selon des critères pragmatiques indiqués plus bas, qui figurent dans la brochure du cours et qui sont indiqués en anglais.

$7 \quad$ La revue de presse est l'activité de démarrage systématique du début de cours. Tout l'espace de la classe est utilisé, afin que les binômes puissent s'isoler (cf. figure 1). J'encourage en particulier l'utilisation de l'espace autour du bureau de l'enseignant et du tableau, afin de le désacraliser et de faciliter les présentations orales ultérieures. Les étudiants utilisent souvent une posture semi-appuyée sur les tables ou contre les murs, ce que j'encourage aussi. La consigne essentielle est l'interdiction de travailler assis, qui rencontre résistance et incrédulité dans les premières semaines. 
Figure 1. Photographie d'une séance de revue de presse : les étudiants échangent, debout, sur des questions d'actualité qu'ils ont choisies eux-mêmes

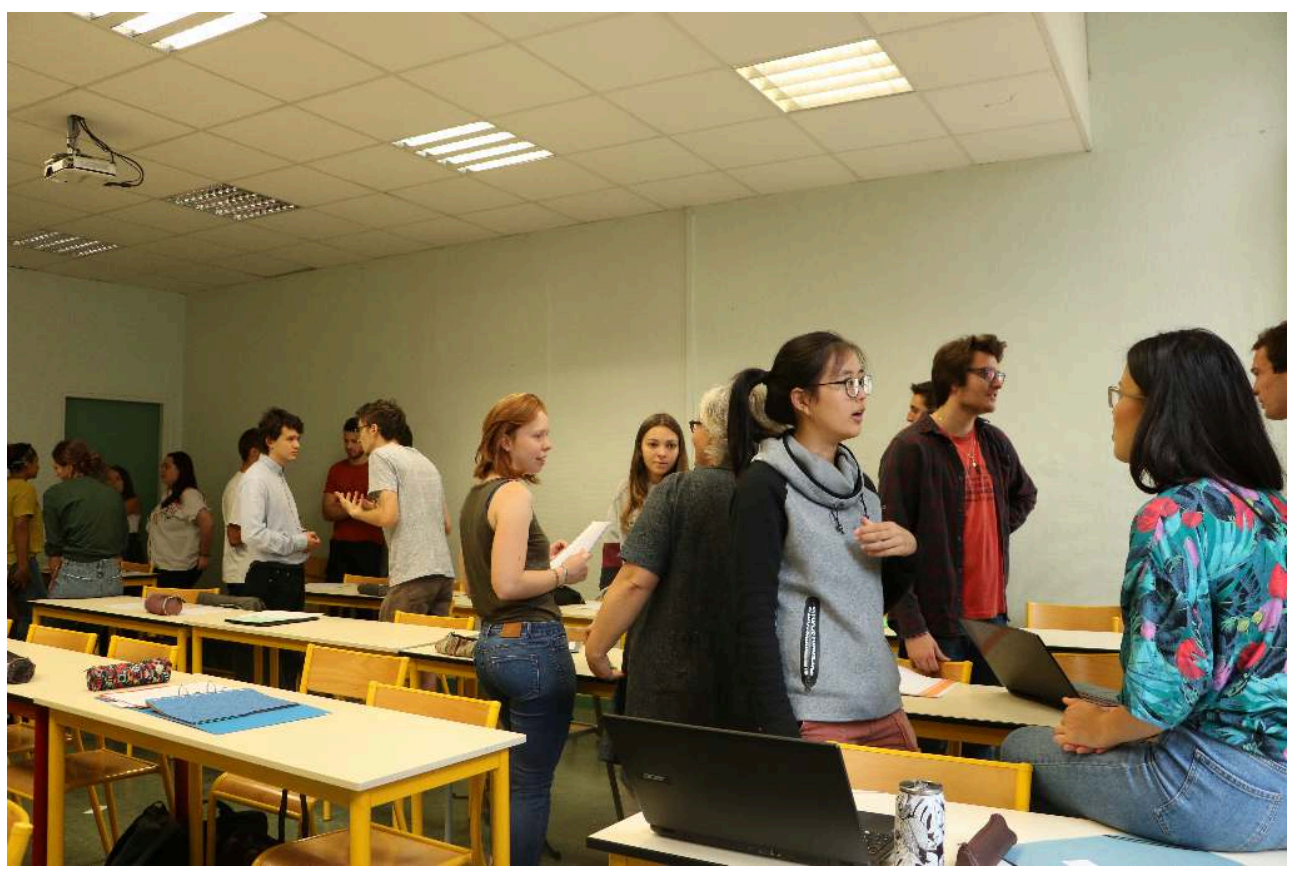

Il est utile de prévoir une dizaine de minutes de reprise au tableau pour éviter la prise de notes pendant l'exercice. Les catégories utilisées pour le feedback peuvent porter sur des structures orales fréquentes, des erreurs fréquemment entendues, de brefs points de grammaire ou le vocabulaire portant sur un thème. C'est notamment l'occasion de mettre en discussion la notion d'utilité du vocabulaire, qui varie dans des groupes hétérogènes. Sur un semestre, les étudiants prépareront 5 articles et écouteront 15 présentations.

9 Les consignes données aux étudiants sont les suivantes :

- Choisissez un article de la presse anglo-saxonne de la semaine

- Expliquez le contenu de votre article oralement (notes non rédigées)

- Amenez à une discussion sur le thème

- Donnez à votre partenaire 3-5 mots ou expressions utiles

- Restez debout

- Parlez à voix haute

- Vous changerez de partenaire après 7 à 10 minutes, deux fois

- Pour la troisième répétition, vous n'avez pas droit à des notes.

10 L'évaluation se fait selon les critères suivants, que nous reprenons ici tels qu'ils sont transmis aux étudiants, c'est-à-dire en anglais :

1. no preparation

2. some preparation but very little transmission

3. preparation but language problems seriously hinder transmission

4. preparation, minor transmission problems, some systematic language errors

5. preparation, minor transmission problems, few or no systematic errors

6. preparation, good transmission and only minor language problems in a rich language context. 


\section{Variantes et prolongements}

- Pour certains groupes très dynamiques, tous les élèves préparent chaque semaine.

- Dans les groupes où beaucoup d'élèves sont faibles, je reprends au tableau quelques structures problématiques et suggère des expressions qui leur font défaut pour communiquer.

- Je fais souvent une reprise au tableau du vocabulaire spécifique de l'actualité de la semaine.

- Pour les groupes très faibles, j'envisage de prévoir 10 minutes de passage à l'écrit individuel pour rédiger un bref compte rendu d'écoute.

- Au bout de 2 semestres, l'évaluation est souvent autoconduite.

- Lorsque le temps et la configuration le permettent, l'activité est pratiquée dans la cour afin de réduire le volume sonore.

- Il est envisageable de faire de la revue de presse spécialisée comme variante au bout de plusieurs semestres. L'objectif n'est alors plus la diversification thématique, mais une meilleure couverture du domaine de spécialité.

\section{Conclusions}

11 Cet exercice permet de répondre à des difficultés identifiées en cours et constitue un outil de coût nul, facile à mettre en place.

\begin{tabular}{|c|c|}
\hline Difficultés identifiées dans mes cours & Apports de la revue de presse \\
\hline Séances trop longues $(2 \mathrm{~h})$ & Segmentation du cours en $20+95$ minutes \\
\hline $\begin{array}{l}\text { Charge cognitive mal répartie (un «bloc» } \\
\text { hebdomadaire sur } 12 \text { semaines) }\end{array}$ & $\begin{array}{l}\text { Préparation à domicile impérative et individuelle, } \\
\text { habitude de lire ou regarder la presse qui perdure } \\
\text { au-delà du semestre }\end{array}$ \\
\hline Inégalités de prise de parole des étudiants & $\begin{array}{l}\text { Travail en binôme: tous doivent parler et écouter } \\
\text { Changement des rôles acquis }\end{array}$ \\
\hline Enseignant auteur et prescripteur du cours & $\begin{array}{l}\text { Transfert de l'impulsion du début de cours vers les } \\
\text { étudiants } \\
\text { Ritualisation du début de cours } \\
\text { Choix des supports par les étudiants }\end{array}$ \\
\hline $\begin{array}{l}\text { Réflexes d'autodéfense et peur du jugement } \\
\text { inhibant l'expression orale }\end{array}$ & $\begin{array}{l}\text { Travail en binôme librement choisi, évaluation des } \\
\text { plus faibles lors de la 3e répétition } \\
\text { Pas de regard du groupe car tous sont immergés } \\
\text { dans un dialogue }\end{array}$ \\
\hline Réticence à la répétition & $\begin{array}{l}\text { Changement de partenaire pour préserver la } \\
\text { dimension communicative }\end{array}$ \\
\hline Refus de prise de parole sans notes & $\begin{array}{l}\text { Deux présentations avec notes avant passage au } \\
\text { spontané }\end{array}$ \\
\hline
\end{tabular}




\begin{tabular}{|l|l|}
\hline $\begin{array}{l}\text { Postures physiques contraintes dans les } \\
\text { présentations orales }\end{array}$ & $\begin{array}{l}\text { Travail debout hors du regard du groupe, } \\
\text { occupation de tout l'espace de la classe à chaque } \\
\text { séance }\end{array}$ \\
\hline Sédentarité et fatigue des étudiants & $\begin{array}{l}\text { Tout l'exercice a lieu debout } \\
\text { Changement de paire toutes les } 7 \text { minutes et } \\
\text { déplacement dans la classe } \\
\text { Mobilité physique, réalisme des postures de prise de } \\
\text { parole }\end{array}$ \\
\hline $\begin{array}{l}\text { Manque de diversité lexicale due à la } \\
\text { spécialisation des cours de LANSAD }\end{array}$ & $\begin{array}{l}\text { Totale liberté des étudiants dans le choix de leur } \\
\text { article } \\
\text { Mission donnée aux étudiants de transmettre du } \\
\text { vocabulaire important } \\
\text { Travail réflexif sur ce qu'est le lexique important } \\
\text { (fréquent/nouveau/rare) }\end{array}$ \\
\hline $\begin{array}{l}\text { Lassitude d'une partie des étudiants par } \\
\text { rapport à l'intégration de leur spécialité dans } \\
\text { les cours de langue }\end{array}$ & $\begin{array}{l}\text { Choix libre des articles } \\
\text { Besoin exprimé de discuter de l'actualité }\end{array}$ \\
\hline
\end{tabular}

12 Cet exercice a changé ma relation avec les étudiants, en réduisant mon temps de parole et en les faisant tous participer. Cela ne signifie pas qu'elle soit toujours appréciée : elle fait peur aux plus faibles au début, frustre ceux qui souhaitent que toute leur production soit écoutée et corrigée, lasse parfois lorsque les sujets sont peu substantiels. Certains étudiants expriment le besoin d'être davantage cadrés, tandis que d'autres plébiscitent l'espace de liberté et la diversification thématique. Les collègues, chargés de cours et lecteurs qui utilisent cette activité font un retour généralement positif, bien que certains soient déroutés par la gestion de groupe pendant les premières semaines. Lorsque l'enseignant a expérimenté l'évolution produite par le changement de positionnement des étudiants, l'inconfort des premières semaines ne pose plus de problème.

13 La prise de parole en continu, telle qu'évaluée formellement au bout de plusieurs semestres (présentations orales ou interactions évaluées intégralement en L3, en M1 et en M2) est bien améliorée, avec en particulier une nette réduction du groupe des étudiants rencontrant de très grandes difficultés à prendre la parole. On ne note pas d'amélioration syntaxique, mais une plus grande fluidité ainsi qu'un lexique plus étendu.

14 La revue de presse ne va pas sans poser quelques difficultés, en particulier le niveau sonore élevé qui, s'il protège l'intimité des binômes, fatigue enseignant et étudiants. Un autre point discutable serait la tendance de certains étudiants à choisir des articles portant sur des sujets très répétitifs ou pouvant sembler superficiels pour des étudiants du supérieur. Par ailleurs, les échanges entre apprenants fournissent peu de correction aux problèmes linguistiques rencontrés, ce qui n'est pas une attente mais qui est parfois identifié comme un manque lors des sondages de retour sur les modules. La rétroaction existe spontanément dans certains binômes, de même que la discussion 
autour de la justesse des énoncés, mais elle n'est pas systématisable sans introduire une primauté de l'étudiant le plus avancé, ce qui nuirait à l'objectif.

L'exercice a cependant révélé d'heureuses surprises : les étudiants posent beaucoup de questions lorsqu'ils repèrent une difficulté qu'ils ne peuvent résoudre en binôme; le respect mutuel des étudiants s'est trouvé renforcé par la révélation des centres d'intérêt et des qualités argumentatives d'étudiants habituellement muets; les étudiants très à l'aise en langue ont changé de statut en devenant facilitateurs au sein des binômes, et en ayant l'occasion de manifester leur intérêt pour des arguments formulés péniblement, grâce à une relative intimité; le recours à des solutions pragmatiques, en particulier une gestuelle de communication plus spontanée ; certains choix thématiques provocateurs ou polémiques amènent de la pluralité et forcent à un débat courtois; la posture modifiée de l'enseignant qui n'a pas la parole recentre le cours sur les étudiants.

Il est à noter que les critères d'évaluation sont modulables selon le niveau de départ des étudiants et le type de formation. La moyenne, dans l'exemple proposé, admet des erreurs de langue systématiques si l'objectif de transmission est atteint, ce qui reflète souvent le profil LANSAD en licence. L'évaluation de la revue de presse relève partiellement de la pièce de théâtre : pour que les étudiants fassent le travail avec régularité, particulièrement en début de licence, la note remplit une fonction de motivation mais elle est peu fiable, car l'enseignant évalue très vite et sur un échantillon. Le fait de ne garder que la note la plus haute du semestre correspond à l'idée qu'on veut ne garder que le meilleur de ce dont ils sont capables, et que l'on prend en compte les baisses de forme et la variabilité de charge de travail dans les matières disciplinaires. La revue de presse ne se conçoit donc que comme une partie de l'évaluation du semestre.

17 L'apport essentiel de cet exercice réside dans la place donnée à la liberté dans le début du cours. De nombreux étudiants arrivent à l'université avec une demande de consignes exactes et craignent plus que tout de déroger à des règles. Cela entrave leur capacité linguistique dans la mesure où le cours de langue est centré sur «la bonne réponse ", censément fournie par l'enseignant. La discussion au sein du binôme doit rester libre. Il est crucial de parvenir à des désaccords, des contradictions, car il n'y a pas de prise de parole sans subjectivité. Parmi les résistances initiales à la revue de presse, on trouve la position debout et le fait de devoir prendre un risque en opérant un premier choix, celui du document, puis un second, qui est l'angle d'approche de la discussion. Ces réticences paraissent corrélées au niveau de langue de l'étudiant, ce qui pourrait constituer un objet d'études. Lorsqu'elles sont levées, le cours entier se trouve enrichi par ce que l'anglais nomme « civil conversation».

\section{NOTES}

1. Une liste indicative de liens vers des médias anglophones du monde entier est mise à disposition sur Moodle. Aucune indication n'est donnée sur la longueur de l'article ni sur le 
thème, de façon à encourager les étudiants à opérer des choix. Les étudiants sont encouragés à choisir des articles récents.

INDEX

Keywords : human science, press review, teaching practices

Mots-clés : compte rendu d'expérience, revue de presse, sciences humaines

\section{AUTEUR}

\section{BÉNÉDICTE REYSSAT}

Bénédicte Reyssat enseigne au Centre Polyglotte de l'UFR Sciences du langage, de l'homme et de la société (SLHS) de l'Université de Franche-Comté. <benedicte.reyssat@univ-fcomte.fr> 